\title{
RESULTS OF TREATMENT OF HOSPITAL STAPHYLOCOCCAL INFECTIONS WITH METHICILLIN
}

\author{
J. W. Harding, B.A., M.B., B.ChIR. \\ R. E. M. Thompson, M.B., B.S., M.R.C.S., L.R.C.P. \\ Bland-Sutton Institute of Pathology, The Middlesex Hospital, London, W.I
}

Methicillin (sodium 6-[2,6 dimethoxybenzamido] penicillinate monohydrate) has been the first compound of major importance resulting from the isolation of pure 6-amino penicillanic acid by Batchelor, Doyle, Nayler and Rolinson (1959). This new penicillin compound is of great therapeutic importance on account of several valuable properties which it possesses. It is bactericidal (Knox, I960), virtually non-toxic (Brown and Acred, I960), is unaffected by staphylococcal penicillinase (Knox, 1960; Rolinson, Stevens, Batchelor, Wood and Chain, 1960) and is effective against all isolated strains of staphylococci in vitro (Thompson, Harding and Simon, 1960). Furthermore, it is effective against staphylococci in vivo (Douthwaite and Trafford, 1960; Stewart, Nixon, Coles, Kesson, Lawson, Thomas, Mishra, Mitchell, Semmens and Wade, 1960). Most of these findings have been confirmed by workers in Canada (Branch, Rodger, Lee and Power, 1960).

We have had the opportunity of observing the results of treatment with this compound of a number of patients infected with penicillinresistant staphylococci in this hospital.

\section{Selection and Method of Treatment}

The cases were selected because they were infected with penicillin-resistant Staphylococcus pyogenes and all patients received the drug for treatment in dosage of $1.0 \mathrm{~g}$. four-hourly by intramuscular injection as recommended by Knudsen and Rolinson (I960), except where otherwise stated. The series may be conveniently divided into two parts: (a) in-patients in this hospital suffering from severe staphylococcal infections; (b) a group of nurses with boils due to penicillin-resistant staphylococci.

The compound used in the treatment of the early cases was obtained from Beecham Research Laboratories Ltd. in solution in $13 \mathrm{ml}$. capped bottles containing 0.5 g. per $\mathrm{ml}$. of the antibiotic. The bottles were stored at $-20^{\circ} \mathrm{C}$. and issued daily for use, during which period storage was at $4^{\circ} \mathrm{C}$. in a domestic refrigerator. The later cases were treated with the commercially available vials of dry substance which were dissolved in $2 \mathrm{ml}$. of sterile distilled water immediately before use.

\section{Case I}

A man, aged 6I, with a long history of peptic ulceration, underwent partial gastrectomy in February, 1960. One week later he developed a wound infection from which Staph. pyogenes (phage type 80) was isolated. The wound broke down rapidly after two days and there was extensive infection in the considerable fatty layer of the anterior abdominal wall. The infection? eventually spread through the fat to the rectus sheath which was visible in the bottom of the wound. During this time the patient was pyrexial up to $103^{\circ} \mathrm{F}$. though the white cell count was only 7,900 per cu. $\mathrm{mm}$. ( $77 \%$ neutrophil polymorphs). A week after the onset of the infection the patient started methicillin. Within $24 \mathrm{hr}$. he was apyrexial and the region around the rectus sheath appeared quite healthy. The wound healed only slowly and the antibiotic was stopped on the seventh day and, although daily wound swabs showed a scanty growth of Staph. pyogenes (type 80), the patient was now both ambulant and feeling well. On the evening of the day on which treatment was stopped, the patient suddenly collapsed and died as a result of pulmonary embolism. A post mortem examination was not performed.

Bacteriologically, the result in this case was poor. Whilst in himself the patient felt better and was able to get up and about, it was disquieting to find the organism still present in the wound on the seventh day of treatment. In the light of subsequent experience with this antibiotic, it would appear likely that the infection, residing largely in fatty tissues with a poor blood supply, could not readily be reached by bactericidal concentrations of the antibiotic. This hypothesis is borne out by the fact that the blood levels of methicillin in this case were $24 \mu \mathrm{g}$. and $3 \mu \mathrm{g}$. per ml. respectively half an hour and $4 \mathrm{hr}$ after a dose of the antibiotic. In this case, a severe 
infection causing wound breakdown on an extensive scale was adequately controlled and healing had commenced to a marked degree when an unfortunate catastrophe terminated further observation.

\section{Case 2}

A man, aged 72, weighing less than 8 st. 7 lb., developed a severe staphylococcal infection (type 80) in the perineal wound seven days after an abdominoperineal excision of the rectum for carcinoma. The wound was discharging several ounces of thick pus daily, there was pyrexia up to ror ${ }^{\circ} \mathrm{F}$. and the white cell count was 14,000 per cu. $\mathrm{mm}$. (83\% neutrophil polymorphs). This infection did not respond to treatment with the usual antibiotics and on the 14th post-operative day he commenced a seven-day course of methicillin. After $36 \mathrm{hr}$. this was given intravenously in $10 \mathrm{ml}$. saline since the thinness and poor musculature of the patient was responsible for much pain at the sites of injection.

Improvement was dramatic; the wound ceased to discharge large quantities of pus and healed rapidly. Three days after commencing treatment the patient was able to sit in a chair without pain. Although, after seven days treatment, a few staphylococci could still be cultured from the wound, in view of the difficulties of intravenous administration of the antibiotic four-hourly and the remarkable improvement both in the infection under treatment and the patient's morale, it was decided to stop the antibiotic and recommence it only if the infection recurred. Two weeks after commencing treatment the patient was discharged to a convalescent home. When subsequently seen as an out-patient, the wound had healed completely and the patient was quite well.

\section{Case 3}

A man, aged 69, was admitted on March 18 with a five-year history of generalized erythroderma. He was being treated with prednisone $40 \mathrm{mg}$. daily and he had secondary skin sepsis associated with recurrent boils. During his stay in hospital he developed multiple skin abscesses on the legs, trunk and buttocks associated with septicamia and septic arthritis of the right wrist. During April and early May Staph. pyogenes (type 80) was isolated from various boils, the urine, the wrist joint and, on May 17, from two blood cultures; all isolations of this organism were resistant to penicillin, streptomycin and tetracycline, but sensitive to chloramphenicol and erythromycin. Over this period he was treated with one or the other of these antibiotics or both simultaneously, but his temperature was raised to about $100^{\circ} \mathrm{F}$. at times during treatment and even higher if one or other of the antibiotics was not used. White cell counts during this period were persistently raised. On May 23 the patient commenced a 30-day course of methicillin and the temperature gradually settled. No further boils appeared after commencing treatment and all subsequent blood cultures were sterile; the white blood count fell to within normal limits and $\mathrm{X}$-ray examination of the right wrist showed no extension of the bone destruction since the beginning of treatment. The patient was delighted with the result of his treatment which coincided with a marked improvement in the skin condition. He was discharged home on June 22, but subsequently died in another hospital a few days later as a result of a severe, and totally unexpected, hæmatemesis.
The unfortunate outcome of this case must not overshadow the remarkable improvement brought $\varrho$ about by methicillin. During hospitalization, and indeed at the commencement of therapy with methicillin, this patient was having large doses of prednisone which is known to potentiate infective $\stackrel{\vec{\rho}}{\stackrel{9}{9}}$ processes, though during the course of the anti- $\bar{C}$ biotic this was gradually reduced to $25 \mathrm{mg}$. daily. 을 During the entire 30 -day course, methicillin was

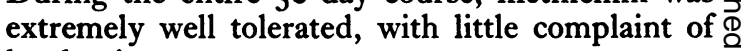
local pain.

\section{Case 4}

A woman of 49 was admitted with mitral stenosis and a pyrexia of unknown origin for investigation to $\vec{\omega}$ exclude subacute bacterial endocarditis. She also gave $\frac{\rho}{8}$ a history of recurrent boils in both groins and on the buttocks for ro weeks. These had been treated by her own 3 doctor with antibiotics and had given rise to much pain and severe constitutional disturbance. On one occasion. such an infection was associated with general malaise and a pyrexia of $104^{\circ} \mathrm{F}$.

During her admission no evidence of bacterial endo- $\vec{c}$ carditis was found, but a swab from a discharging boil of in the groin yielded a pure culture of penicillin-resistant $ᄋ$ staphylococci (type 80/81). Methicillin was administered for seven days and the patient complained of severe pain at the site of injection. She was discharged two weeks after starting treatment and when last seen two months later she was quite well, and had had no further boils or other infective foci.

\section{Case 5}

A woman of 42 with severe rheumatoid arthritis wh admitted for investigation of sudden loss of weight associated with peripheral neuritis and right foot-drop. On January 12, 1960, she developed pneumonia and Staph. pyogenes (type 80) sensitive to chloramphenicol and erythromycin, resistant to penicillin, streptomycin $\mathbb{Q}$ and tetracycline, was isolated from the sputum. She was treated with both chloramphenicol and erythro- $\overrightarrow{\overline{0}}$ mycin simultaneously and subsequently developed an abscess in the apical segment of the right lower lobe. The patient failed to respond to the antibiotic therapy and on January 23 Staph. pyogenes, with identical antibiotic sensitivities, was again isolated in profusion from the sputum.

On January 27 the patient commenced methicillin, coincident with the onset of abdominal pain. On the next day laparotomy was performed and a gangrenous $\delta$ patch of crecum was found which, in view of the patient's critical condition, was invaginated into the creum and oversewn with omentum. A caval drip was set up and $\frac{}{2}$ methicillin was given I g. four-hourly in $10 \mathrm{ml}$. saline $D$ into the drip. Culture of the sputum on January 30 을 and subsequently yielded no staphylococci. In view of the lung abscess, methicillin was continued for six $\mathrm{N}$ weeks. Serial chest $\mathrm{X}$-rays showed gradual improvement over this period.

During March and April the condition of the patient gradually deteriorated. She had recurrent attacks of bronchopneumonia during which a variety of organisms were isolated, most frequently Bacterium coli. During May she became increasingly breathless and eventually died on the $25^{\text {th }}$ of that month.

In this case methicillin was used on a critically ill, grossly debilitated patient who $3^{6} \mathrm{hr}$. after 


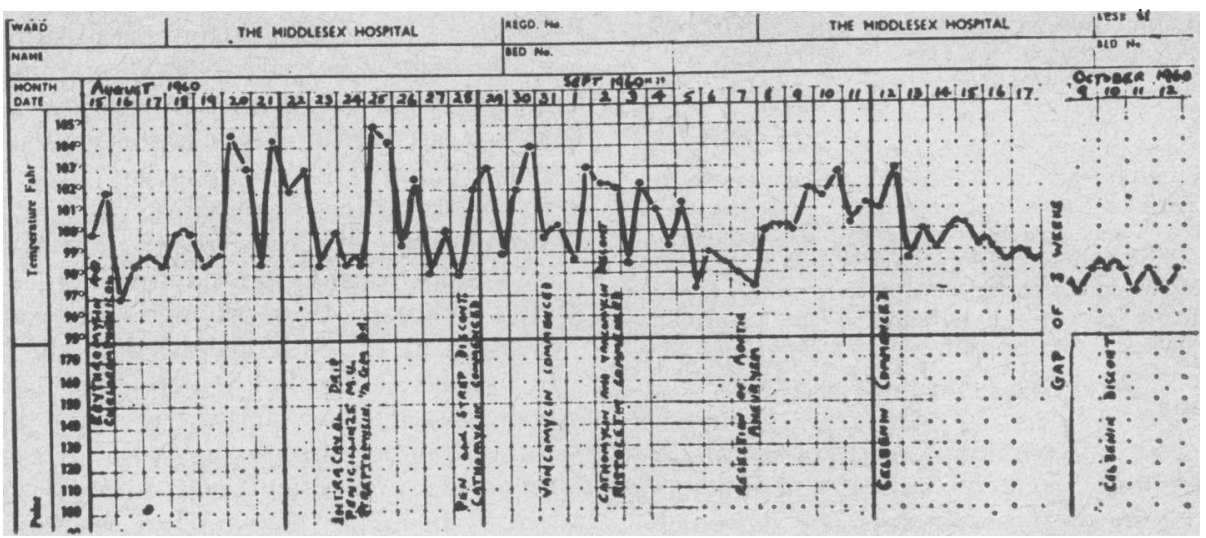

Fig. 1.-Showing the effect of Celbenin and other antibiotics on the progress of Case 7.

starting treatment had to undergo an abdominal operation under general anæsthesia. Though the patient died eventually from cachexia and bronchopneumonia, in view of the elimination of staphylococci from the sputum and the survival of the hazards of laparotomy and general anæsthetic whilst such a virulent infection was present in the chest, methicillin must be judged successful in the treatment of this case.

\section{Case 6}

A woman, aged 47 , previously under out-patient medical treatment for chronic bronchitis and asthma, was admitted with a six-week history of pain in the right thigh, on May 25. X-ray showed an area of bone destruction in the right upper femur which on exploration was shown to be osteomyelitis; culture from this lesion revealed penicillin-resistant staphylococci (type 80).

On admission the patient had a temperature of ror. $2^{\circ} \mathrm{F}$. and a total leucocyte count of $14,35^{\circ}$. On May 30 a course of methicillin was commenced and this was terminated on June 30,1960 . On June 8 the drain was removed from a dry sterile wound. Clinically, the patient made a steady recovery and was discharged on July 25 to a convalescent home. When seen at the end of October, the patient was weight-bearing on the affected leg and very well.

\section{Case 7}

A little girl aged six and weighing only 2 st. $4 \mathrm{lb}$. was admitted to hospital on July 29. In April she had had a patent ductus arteriosus ligated in Calcutta and subsequently suffered from a Staph. pyogenes endocarditis. It was at this time thought that the ductus arteriosus had recanalized. Subsequent exploration showed the ductus arteriosus intact, but an aneurysm was found in the region of the pulmonary trunk and extending towards the left subclavian artery. A flare-up of the endocarditis followed this thoracotomy and a strain of Staph. pyogenes (resistant to penicillin, streptomycin and terramycin; sensitive to aureomycin, achromycin and chloramphenicol) was isolated. The child was then transferred to this country for treatment.

On admission there was a pyrexia of up to $102^{\circ} \mathrm{F}$. uncontrolled by erythromycin and chloramphenicol. Later, various other antibiotics were administered without effect (see Fig. I) and up till this time no organism had been isolated in this hospital from the patient. On September 7 an aneurysm of the ductus area and aorta was resected under hypothermia. The wall of this aneurysm was necrotic and infected and from it Staph. pyogenes (type 52/80/8I) was isolated. Post-operatively, pyrexia continued and methicillin was first given on September 12 at a dosage rate of $1 \mathrm{~g}$. four-hourly. As shown in Fig. I the temperature gradually fell to normal. This patient was fully ambulant and recovery from the infection appeared complete. Subsequently the child showed signs of increasing heart failure and died on November 7. At post mortem there was no sign of active staphylococcal infection and attempts to isolate the organism failed.

\section{Case 8}

A man of 46 developed a penicillin-resistant staphylococcal (type 52A) bronchopneumonia following closure of an atrial septal defect. This infection was treated originally with penicillin and later with chloramphenicol and erythromycin without success. Treatment was started with methicillin on October 15 and continued for seven days. At no time after commencing methicillin was Staph. pyogenes isolated from the sputum and the patient made an uninterrupted recovery.

\section{Case 9}

A nurse aged 20 was admitted to hospital on August 2 with a swollen, painful, almost closed left eye due to acute frontal sinusitis. Pus from the left nostril was cultured and a course of tetracycline combined with local therapy to the nose in the form of drops and inhalations was commenced.

Culture of the nasal pus yielded pure Staph. pyogenes (type 80/81) resistant to penicillin, streptomycin and tetracycline. In view of this a seven-day course of methicillin was substituted for tetracycline on August 4 and the patient's condition rapidly improved. In three days the temperature was normal and the swelling had subsided. The patient was discharged fit and well on August 15 .

\section{Case 10}

A nurse suffering from recurrent axillary boils due to penicillin-resistant staphylococci (type 80) was treated with methicillin for five days in June 1960. The infection cleared up rapidly and the patient was free 
from trouble for three months until September 1960 when the axillary furunculosis (type $80 / 8 \mathrm{I}$ ) recurred.

\section{Case II}

In August a young nurse with a carbuncle of buttock due to a penicillin-resistant staphylococcus (type 8o/8r) associated with pyrexia, surrounding cellulitis and general malaise, was treated with a five-day course of methicillin. The lesion healed rapidly and so marked was the improvement that on completion of the course she was able to leave for a holiday on the Continent and has remained perfectly well.

\section{Discussion}

Eleven cases of penicillin-resistant staphylococcal infections treated by methicillin are recorded. Some of these patients had extremely serious infections and in all cases methicillin has succeeded in eliminating the infection and, although in four cases the patients treated have subsequently died, in no case can their deaths be attributed either directly to treatment with methicillin or to failure of methicillin to control the penicillin-resistant staphylococcal infection from which they were suffering.

In all cases the antibiotic was well tolerated, but several patients complained of local pain at the site of injection. This symptom was by no means constant. Case 2, who was exceptionally thin, suffered markedly from this and Case 4 was similarly affected. She was an exceptionally good witness who had had in the past several courses of benzyl penicillin which caused negligible discomfort, but she complained bitterly of the marked pain at the site of injection of methicillin. One nurse also complained of pain, and the remainder of the nurses, whilst making no complaint of pain, agreed on questioning that injections of methicillin were more painful than injections of the parent compound, benzyl penicillin. It was notable that the heavier patients had no complaint of pain even on direct questioning.

No toxic manifestations were encountered at any time during or after therapy in any of these cases. Cases 3, 5, 6 and 7 all had prolonged treatment lasting four weeks or more without any ill effect. In Case 7 the dosage (I g. four-hourly for a patient weighing 2 st. $4 \mathrm{lb}$.) was markedly in excess of the recommended levels, but nevertheless the course was completed without any suggestion of toxicity. Case 6 developed eosinophilia during treatment, which rose until the eosinophils represented $63 \%$ of the total white cell count. At this time, it was felt that such a marked eosinophilia represented an allergic reaction to penicillin, but in the absence of any other allergic manifestations, it was decided to complete the course of penicillin since, if it was an allergic response, temporary suspension of the course of the antibiotic for investigation of allergy气 might make it very difficult, if not impossible, to $\frac{\pi}{\bar{\alpha}}$ recommence treatment with methicillin which was so essential to eliminate this patient's infection. Subsequently this patient has bien seen some 16 weeks after termination of the course of methicillin. Her leg is free from infection and she등 is weight-bearing but continues to suffer from $\overline{\overline{\bar{s}}}$. mild asthma with occasional acut 2 exacerbations. $\mathbb{\Phi}$ When seen, the patient showed no allergic reaction to skin testing with methicillin and benzyl ${ }^{\infty}$ penicillin but her peripheral white blood count $\overrightarrow{0}$ showed an eosinophilia of $37 \%$. A case has been $\overrightarrow{ }$ reported of a patient with known sensitivity to $\omega$ benzyl penicillin showing no reaction to methi-D cillin (Branch, Rodger, Lee and Power, r 1960).3

An observation of great personal interest to usw was the recurrence of axillary infection $2 \frac{1}{2}$ months ${ }^{-}$ after treatment in Case 10. The treatment of ${ }_{0}$ recurrent axillary boils is a great problem in both: general practice and casualty departments through-co out the country. In many cases an isolated boil음 may occur which is quite adequately and simply treated by incision and drainage with or without antibiotic therapy as indicated clinically, and often recurrent axillary boils are similarly treated as if $\stackrel{\text { ? }}{+}$ they were single non-recurring lesions. It must $\vec{\omega}$ be realized, however, that such treatment $\oint_{s}$. aimed at removing and eliminating the painfa focus of infection and at the same time it makes absolutely no attempt to treat or control any underlying condition which may be giving rise to the recurrent furunculosis. Such treatment is $\frac{0}{\varnothing}$ usually unsuccessful and, if the condition persists, $\stackrel{\Omega}{\rightarrow}$ too often the patient is condemned to a ritual of $\bar{O}$ taking a variety of antibiotics punctuated by 3 incision and drainage of the recurrent boils. The efficacy of any treatment aimed at eliminating axillary infection is most difficult to assess; since there is a tendency to spontaneous resolution, the credit for a cure is often given to the last antibiotic 3 . used.

We have found no signs of the staphylococci developing resistance to methicillin during treat- 0 ment with the drug in any of the cases. Indeed, we are of the opinion that the development of $\frac{}{0}$ such resistance is likely to be a rare event and when it does occur will be unlikely to be suf- $\tilde{N}$ ficiently severe or sufficiently stable to jeopardize N the value of the drug in the same way that penicil-N linase-producing strains do benzyl penicillin.

All the cases described have been infected with types of Staph. pyogenes that are found commonly in hospitals $(52 \mathrm{~A} ; 80 ; 80 / 8 \mathrm{I} ; 52 / 80 / 8 \mathrm{I})$ causing $\stackrel{\oplus}{\rightarrow}$ post-operative wound infections and primary 0 staphylococcal infections in patients and staff. $\bar{O}$ We feel that there is a strong case for treating all $\stackrel{\odot}{\stackrel{D}{~}}$ staphylococcal infections in hospitals with methi- $\frac{\mathbb{D}}{\mathbb{D}}$ 
cillin regardless of the antibiotic sensitivities of the infecting organism.

\section{Summary}

I. Eleven cases treated with methicillin are recorded and in all cases staphylococcal infection was eliminated.

2. No case showed any evidence of toxicity due to the drug, although acquired hypersensitivity was suspected in one case.
3. None of the strains of staphylococci showed any evidence of developing resistance to the drug.

We wish to thank the following members of the consultant staff of the Middlesex Hospital: Mr. J. R. Belcher, Dr. F. Ray Bettley, Mr. T. Holmes Sellors, Mr. O. V. Lloyd Davies, Mr. C. J. B. Murray, Mr. P. H. Newman, Dr. F. H. Scadding and Dr. A. Willcox; also the resident medical officer, Dr. A. O. Robson, for permission to use cases under their care and for their help and criticism in the preparation of this paper. We should also like to thank Beecham Research Laboratories Ltd. for generous supplies of 'Celbenin'.

\section{REFERENCES}

Batchelor, F. R., Doyle, F. P., Nayler, J. H. C., and Rolinson, G. N. (1959): Synthesis of penicillin: 6-aminopenicillanic acid in penicillin fermentations, Nature, Lond., 183, 257.

Branch, A., Rodger, K. C., Lee, R. W., and Power, Edna E. (1960): Clinical and Laboratory Experiences with a New Synthetic Penicillin-2, 6-dimethoxyphenol penicillin, Canad. med. Ass. Y., 83, 991.

Brown, D. M., and ACRED, P. (1960): Chemotherapeutic Studies on a New Antibiotic-BRL 1241, Lancet, ii, 568. Douthwaite, A. H., and Trafrord, J. A. P. (1960): A New Synthetic Penicillin, Brit. med. F., ii, 687.

Knox, R. (I960): A New Penicillin (BRL 124I) Active Against Penicillin-resistant Staphylococci, Ibid., ii, 690.

KNUdSEN, E. T., and Rolinson, G. N. (1960): Absorption and Excretion of a New Antibiotic (BRL 1241), Ibid., ii, 700.

Rolinson, G. N., Stevens, Shirley, Batchelor, F. R., Cameron Wood, J., and Chain, E. B. (I960): Bacteriological Studies on a New Penicillin-BRL 1241, Lancet, ii, 564.

Stewart, G. T., Nixon, H. H., Coles, H. M. T., Kesson, C. W., Lawson, D., Thomas, R. G., Mishra, J. N., Mitchell, M. Eileen, Semmens, J. Marjorie, and WAdE, T. H. H. (1960): Report on Clinical Use of BRL

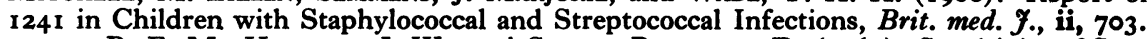

Thompson, R. E. M., Harding, J. W., and Simon, Rosemary D. (1960): Sensitivity of Staphylococcus Pyogenes to Benzylpenicillin and BRL I24I, Ibid., ii, 708. 